\section{A case of anterior cerebral artery A1 segment hypoplasia syndrome presenting with right lower limb monoplegia, abulia, and urinary incontinence}

Sir,

A1 segment of anterior cerebral artery (ACA) is an uncommon site for ischemic strokes and is rarely symptomatic. Here, we describe a case of A1 segment of left ACA hypoplasia, presented with monoplegia of right lower limb, abulia, and bladder incontinence.

A 50-year-old female came to us because of sudden onset of weakness in right lower limb, history of urinary incontinence, and decreased output of words. There was no history of headache, vomiting, vertigo, trauma, seizure, or loss of consciousness and no history suggestive of any chronic illness or addiction. On examination, she was conscious, cooperative and well-oriented with time, place, and person. The blood pressure was $128 / 80 \mathrm{mmHg}$, pulse rate was $84 / \mathrm{min}$ regular and all peripheral pulses were palpable and synchronous, respiratory rate was $16 \mathrm{breath} / \mathrm{min}$, and temperate was $99^{\circ} \mathrm{F}$. Neurological examination revealed decreased output of speech. Her comprehension, understanding and articulation of word were good, but she used to respond after a pause of few seconds and word output was decreased. Cranial nerve 
examination was normal. There was no illusion, delusion, hallucination, and antisocial activity. Tone was decreased in right lower limb with a power of $1 / 5$ in all groups of muscles. Tone and power in all other limbs were normal. Knee and ankle reflex were decreased in the right lower limb. Plantar reflex was bilaterally flexor. Sensory system examination was normal. National health stroke severity score (NIHSS) was 5 . Her respiratory, cardiovascular and gastrointestinal system examinations were within normal limit.

Laboratory examination revealed normal hematology and biochemistry profiles. She tested negative for malaria, hepatitis B surface antigen, hepatitis $C$ virus, and HIV. Cerebrospinal fluid examination was normal. Electrocardiogram, echocardiogram, ultrasonography of abdomen, and chest X-ray were within normal limit. Magnetic resonance imaging (MRI) of the brain showed a medium sized infarct in the left para sagittal frontoparietal brain parenchyma with early hemorrhagic transformation [Figure 1a-d]. In magnetic resonance angiograms (MRA) the A1 segment of left ACA was not visible and indicating hypoplasia. The A2 segment was normal indicting flow via the anterior communicating artery. The right ACA was normal. MR venogram was normal [Figure 2a-d].

ACA is a terminal branch of internal carotid artery and forms the anterior component of the circle of Willis along with the anterior communicating artery. It supplies the orbitofrontal and medial hemispheric portions of the brain including paracentral lobule and the outer upper strip of the frontal lobe. Of the all ischemic strokes ACA territory strokes constitutes only $0.6-3 \%$. Among the variation in the anatomy of the circle of Willis, a small

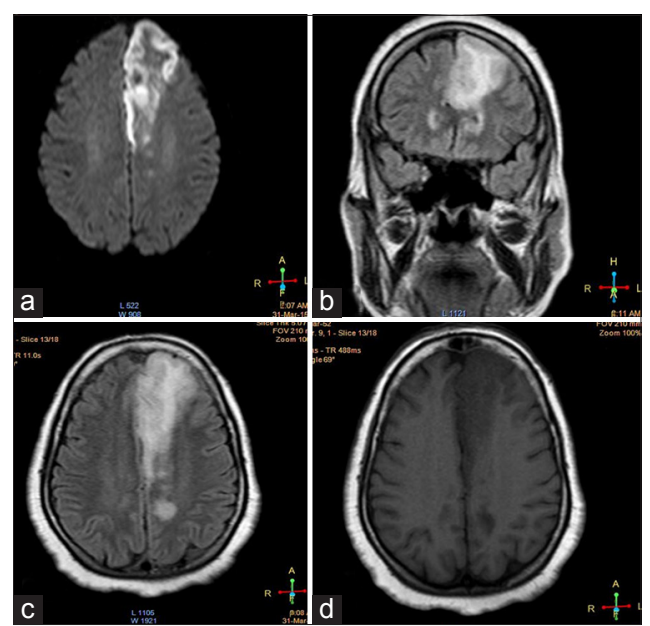

Figure 1: (a) Diffusion-weighted image axial section showing patchy restricted diffusion; (b) fluid-attenuated inversion recovery image coronal section showing hyperintensity with edema; (c) fluid-attenuated inversion recovery image axial section showing hyperintensity with edema; (d) T1 weighted image axial section showing hypointense lesion degree of asymmetry of anterior arteries is commonly found. The variations related with the A1 segment of ACA are agenesis, hypoplasia and duplication. A1 segment hypoplasia is an uncommon fatal variant of the circle of Willis. ${ }^{[1]}$ The frequency of this congenital variation is $1-13 \%$ as derived from angiograms and autopsy reports in various experimental studies. ${ }^{[1,2]}$ The A1 segment of the ACA is a principle supplier of anterior collateral blood flow. This segment is also a source of numerous penetrating striatal arteries that supply the anterior hypothalamus, septum pellucidum, and the anterior and inferior portions of the corpus striatum. A1 segment hypoplasia as a noncompetent part of the circle of Willis appears to be a risk factor for acute ischemic stroke. ${ }^{[1]}$ The majority of these patients have small vessel occlusive strokes, especially within the striatum because of poor collateral capacity that would render arteries penetrating the striatum vulnerable to ischemic attack. ${ }^{[3]}$ There is also poor thromboembolism clearance within the striatum with defective collateral circulation. ${ }^{[3,4]}$ A1 segment hypoplasia related ischemic stroke are usually tolerable, predominantly with sensorimotor deficit contralateral to the A1 segment hypoplasia and with low NIHSS. ${ }^{[1]}$ Patients with such a defect may have intact contralateral collateral circulation, hence more vulnerable for ipsilateral hemispheric ischemic stroke. ${ }^{[5]}$ The A1 hypoplasia is readily detected by MRA and area of acute ischemic lesions can be localized by diffusion-weighted imaging (DWI) within early hours.

The patient presented with acute onset of complete weakness of right lower limb, urinary incontinence, and abulia. MRA of brain vessels and DWI of MRI brain showed hypoplasia of A1 segment of left ACA and fresh infarct in the area of the left superior frontal region. We also

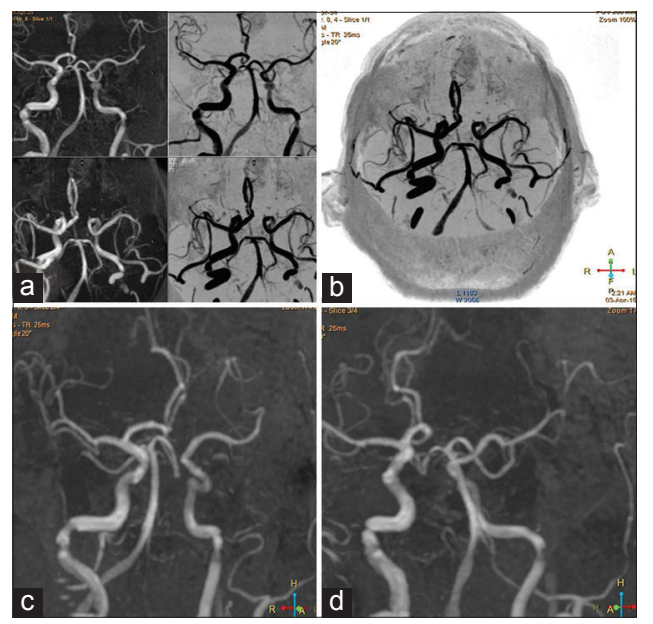

Figure 2: (a-d) The A1 segment of left anterior cerebral artery is not visible indicating hypoplasia. Right anterior cerebral artery is normal. The A2 segment is visible normally indicating flow via the anterior communicating artery. There is no evidence of aneurysm 
conclude that hypoplasia of A1 segment of ACA is a risk factor for development for ipsilateral hemispheric ischemic stroke because the circulation in the contra lateral side is maintained through compensated collateral circulation. Such large area of infarction in the ACA territory presenting with monoplegia, abulia, and urinary incontinence is very rare for ACA A1 segment hypoplasia syndrome.

What could have triggered the brain hypoperfusion at the time of onset is difficult to access, as the patient when seen by us did not have any cause to suggest hypoperfusion. The only thing that we can presume is the presence of some transient arrhythmia leading to hypoperfusion, which by the time the patient was attended had recovered.

\section{Financial support and sponsorship}

Nil.

\section{Conflicts of interest}

There are no conflicts of interest.

Manoj Lakhotia, Hans Raj Pahadiya, Gopal Raj Prajapati, Akanksha Choudhary, Ronak Gandhi, Hemant Jangid ${ }^{1}$

Department of Medicine, Dr. S. N. Medical College, ${ }^{1}$ Department Radiodiagnosis, Satyam MRI Centre, Dr. S. N. Medical College, Jodhpur, Rajasthan, India

Address for correspondence: Dr. Hans Raj Pahadiya, Department of Medicine, Dr. S. N. Medical College, Jodhpur - 342 001, Rajasthan, India. E-mail: drhans05sms@gmail.com

\section{References}

1. Chuang YM, Liu CY, Pan PJ, Lin CP. Anterior cerebral artery A1 segment hypoplasia may contribute to A1 hypoplasia syndrome. Eur Neurol 2007;57:208-11.

2. Mäurer J, Mäurer E, Perneczky A. Surgically verified variations in the A1 segment of the anterior cerebral artery. Report of two cases. J Neurosurg 1991;75:950-3.

3. Kang DW, Chu K, Ko SB, Kwon SJ, Yoon BW, Roh JK. Lesion patterns and mechanism of ischemia in internal carotid artery disease: A diffusion-weighted imaging study. Arch Neurol 2002;59:1577-82.

4. Wang CX, Todd KG, Yang Y, Gordon T, Shuaib A. Patency of cerebral microvessels after focal embolic stroke in the rat. J Cereb Blood Flow Metab 2001;21:413-21.

5. van Everdingen KJ, Visser GH, Klijn CJ, Kappelle LJ, van der Grond J. Role of collateral flow on cerebral hemodynamics in patients with unilateral internal carotid artery occlusion. Ann Neurol 1998;44:167-76.

This is an open access article distributed under the terms of the Creative Commons Attribution-NonCommercial-ShareAlike 3.0 License, which allows others to remix, tweak, and build upon the work non-commercially, as long as the author is credited and the new creations are licensed under the identical terms.

\begin{tabular}{|l|l|}
\hline \multicolumn{2}{|c|}{ Access this article online } \\
\hline Quick Response Code: & Website: \\
\hline & www.ruralneuropractice.com \\
\cline { 2 - 2 } & \\
\hline
\end{tabular}

How to cite this article: Lakhotia M, Pahadiya HR, Prajapati GR, Choudhary A, Gandhi R, Jangid H. A case of anterior cerebral artery A1 segment hypoplasia syndrome presenting with right lower limb monoplegia, abulia, and urinary incontinence. J Neurosci Rural Pract 2016;7:189-91. 